\title{
A Case of Recurrent Pulmonary Inflammatory Myofibroblastic Tumor with Aggressive Metastasis after Complete Resection
}

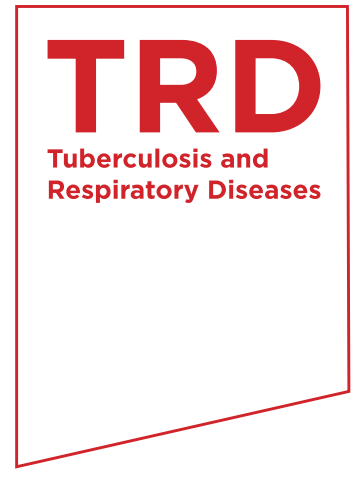

\author{
Chae Ho Moon, M.D., Jong Ho Yoon, M.D., Geon Wook Kang, M.D., Seong Hyeon Lee, M.D., Jeong \\ Su Baek, M.D., Seo Yun Kim, M.D., Hye-Ryoun Kim, M.D. and Cheol Hyeon Kim, M.D. \\ Department of Internal Medicine, Korea Cancer Center Hospital, Korea Institute of Radiological and Medical Sciences, Seoul, \\ Korea
}

An inflammatory myofibroblastic tumor (IMT) is a rare disease entity reported to arise in various organs. It is thought to be a neoplastic or reactive inflammatory condition, controversially. The treatment of choice for myofibroblastic tumor is surgery, and recurrence is known to be rare. The optimal treatment method is not well-known for patients ineligible for surgery. We report a 47-year-old patient with aggressive recurrent IMT of the lungs. The patient had been admitted for an evaluation of back-pain two years after a complete resection of pulmonary IMT. Radiation therapy was performed for multiple bone recurrences, and the symptoms were improved. However the patient presented again with aggravated back-pain six months later. High-dose steroid and non-steroidal anti-inflammatory drugs were administered, but the disease progressed aggressively, resulting in spinal cord compression and metastasis to intra-abdominal organs. This is a very rare case of aggressively recurrent pulmonary IMT with multi-organ metastasis.

Keywords: Lung Neoplasms; Myofibroblasts; Lung; Recurrence; Neoplasm Metastasis

\section{Introduction}

An inflammatory myofibroblastic tumor (IMT) is a very rare disease entity reported to arise in various organs within

Address for correspondence: Cheol Hyeon Kim, M.D.

Department of Internal Medicine, Korea Cancer Center Hospital, Korea Institute of Radiological and Medical Sciences, 75 Nowon-ro, Nowon-gu, Seoul 139-706, Korea

Phone: 82-2-970-1209, Fax: 82-2-970-2438

E-mail: cheol@kcch.re.kr

Received: Jun. 18, 2013

Revised: Jul. 26, 2013

Accepted: Aug. 1, 2013

(c) It is identical to the Creative Commons Attribution Non-Commercial License (http://creativecommons.org/licenses/by-nc/3.0/).

Copyright (c) 2013

The Korean Academy of Tuberculosis and Respiratory Diseases. All rights reserved. the abdomen but is usually arising in the lungs. An IMT of the lungs is known to be approximately $0.04-1 \%$ of all lung tumors, and can arise in any age group, but is mainly common below 40 years of age with the highest onset rate in children below 16 years ${ }^{1}$. IMT is characterized by spindle cell proliferation without the evidence of atypia, pathologically showing the differentiation of fibroblast or myofibroblast in the form of small bundles or spirals, and is accompanied by the infiltration of inflammatory cells.

Most IMT of the lungs is usually treated by surgery, and is known to have good prognosis in case of complete excision ${ }^{1}$. Patients with recurrent IMT of the lungs have rarely been reported and no effective treatment for such disease has been established. In the case of a recurrence in which it is impossible to perform an excision, if it originated within the abdominal cavity, there were some cases which showed a response to anticancer treatment ${ }^{2,3}$. However, in the case of IMT observed in the bones, there were rare cases reported to be responsive to anticancer treatment, steroid administration, radiotherapy, 
and combined therapy of non-steroidal anti-inflammatory drugs $^{2,4}$. The authors report a rare case of a very aggressive recurrent IMT of the lungs after complete resection and which showed a metastasis within the bones and abdominal cavity unresponsive to treatment. Written informed consent was obtained from the patient's family for publication of this case report and accompanying images.

\section{Case Report}

\section{Present illness}

A 47-year-old male patient was referred to our hospital. The patient had discovered a shadow in the upper lung field from a chest X-ray during a medical check-up at an outside hospital on April 2009. A 30-mm sized well-defined low attenuation lesion was observed in the left upper lung on a chest radiography and a computed tomography (CT) (Figure 1). Positron emission tomography/computed tomography (PET/CT) showed maximum standardized uptake values (SUV) of 10 in the masses in the left upper lung. Bronchoscopy revealed a whitish tumor obstructing the anterior segmental bronchus of left upper lung upper division covered with necrotic material, and biopsy specimens of the tumor was taken during the bronchoscopic examination, and was reported to be spindle cell proliferation according to pathological findings. A radical left upper lobectomy was performed on July 2009 for the purpose of diagnosis and treatment, and an IMT was confirmed by histopathology. On gross examination, the tumor was a single mass with a regular border and adjacent to the bronchus, localized in the lung parenchyma and measured $4.3 \times 3.5$ $\mathrm{cm}$ (Figure 2A). Microscopic examination revealed spindle cells proliferation without atypia and inflammatory cells (Figure 2B). According to immunohistochemical examination, the myofibroblasts were negative for all cytokeratin, smooth muscle actin, desmin, and Bcl-2. In addition, the myofibroblasts were focally positive for anaplastic lymphoma kinase-1
(ALK1) (Figure 2C). Fluorescence in situ hybridization of $A L K$ rearrangements was not done because of the patient's disagreement. There was no evidence of metastasis with examination of 32 lymph nodes. On postoperative outpatient follow-up, the patient complained of back pain on August 2011, approximately 2 years afterwards. While there was no evidence of recurrence in the lungs from the chest CT performed then, the PET/CT showed multiple bone metastasis (right third rib [SUV, 7.8], thoracic 12th spine [SUV, 10.2], and left sacroiliac bone [SUV, 12.9]) (Figure 3A). According to the biopsy performed on the thoracic spine, a recurrence of the IMT was confirmed. The patient received a total of $36 \mathrm{~Gy}$ focal irradiation on the thoracic spine to control the back pain, and was discharged after the pain was alleviated. Afterwards, the back pain aggravated in just 6 months and therefore, the patient made a visit.

\section{Past medical history, family history and social history}

The patient had no specific past medical history and family history. He has a history of a pack of cigarette smoking for 15 years and quit smoking in 2005. There was no other remarkability.

\section{Physical examination}

According to chest and abdominal check-up, no abnormality was observed, and the patient complained of pain in the left pelvis and pain in the left calf. There were no other specific findings, and there was no clearly palpable tumor.

\section{Laboratory findings}

According to the blood test, the white blood cell count increased to $17,400 / \mathrm{mm}^{3}$, the hemoglobin count was $13.6 \mathrm{~g} / \mathrm{dL}$, and platelet was $274,000 / \mathrm{mm}^{3}$, which is within normal range. The blood urea nitrogen, creatinine, liver function test, and electrolyte result were within normal range, and there was a
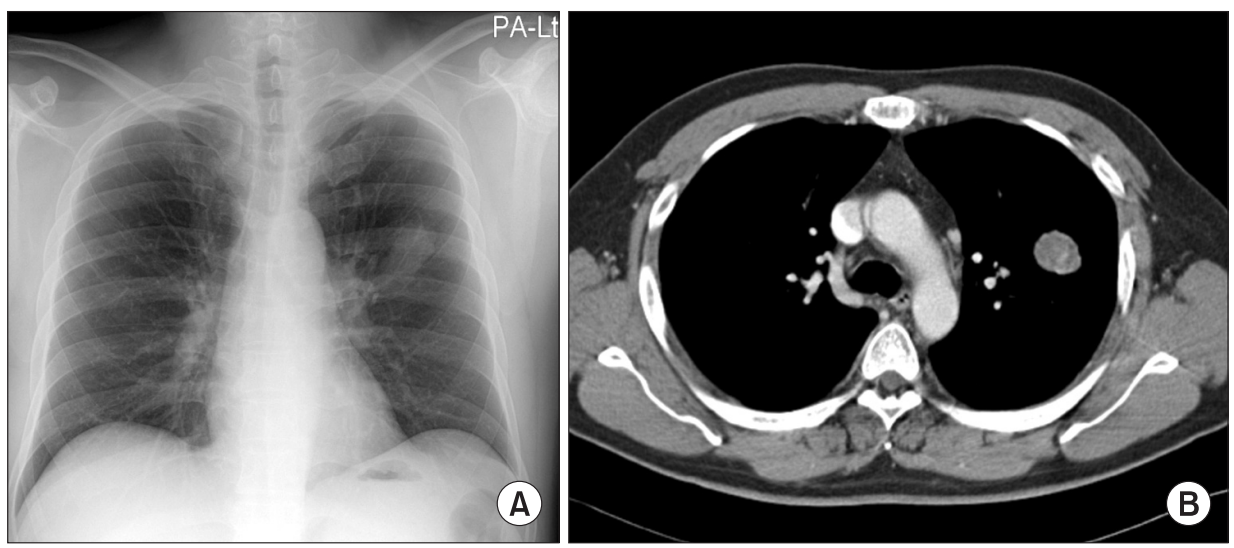

Figure 1. Simple chest radiography (A) and contrast-enhanced computed tomography (CT) (B) taken before the operation. Chest CT shows a 3-cm sized, well-marginated oval mass in the left upper lobe of lung. 

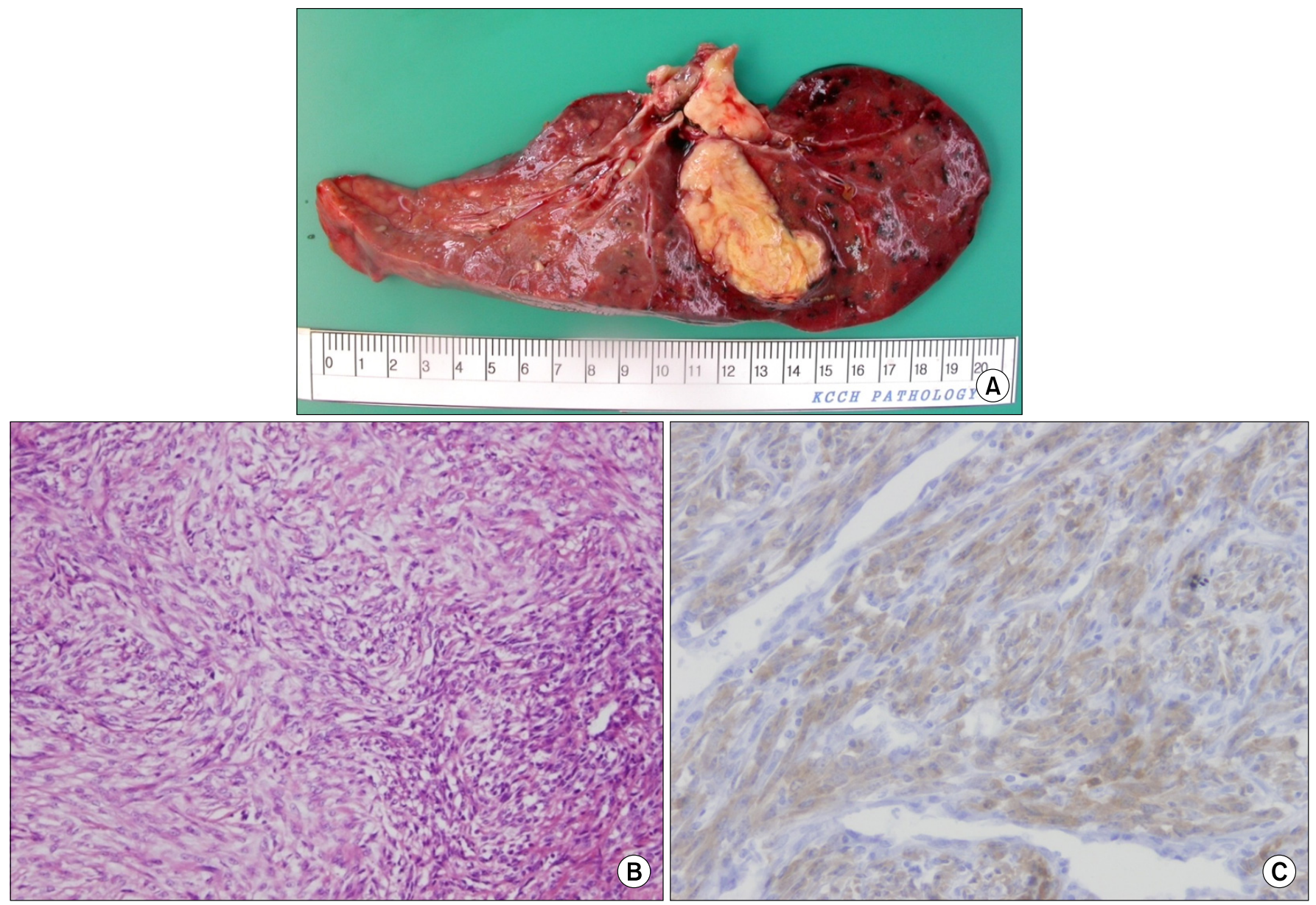

Figure 2. Pathologic findings of resected specimens from the patient. (A) Grossly, there is a solitary mass with a yellowish-gray color and rubbery consistency. (B) Tumor contains a mixture of spindle cells and infiltrating inflammatory cells containing lymphocytes and plasma cells (H\&E stain, $\times 500$ ). (C) Immunohistochemical staining result for anaplastic lymphoma kinase-1 shows focal positivity in cytoplasm of tumor cells $(\times 1,000)$.
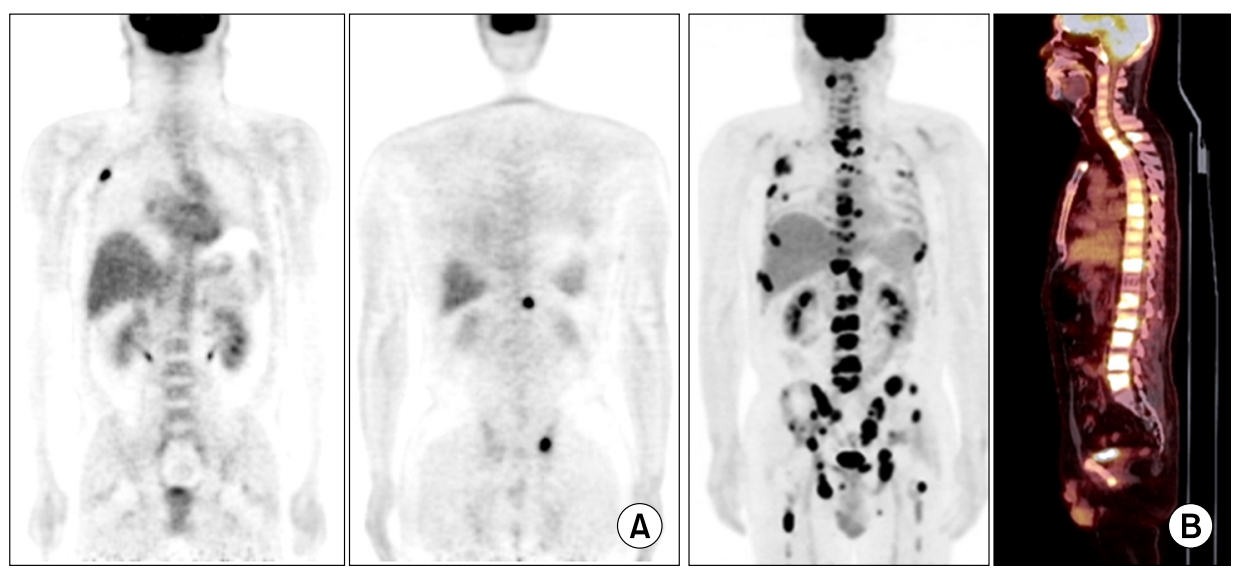

Figure 3. (A) Fluorodeoxyglucose positron emission tomography/computed tomography (PET/CT) images obtained before the first radiation therapy. PET/CT shows abnormal metabolic activity with a high standardized uptake value in the right 3rd rib, the thoracic 12th vertebra and the left sacroiliac bone. (B) PET/CT performed before the second radiation therapy. PET/CT shows remarkably increased number of metastatic lesions with increased fluorodeoxyglucose uptake at multiple bones during the time interval.

slight increase in C-reactive protein with $1.36 \mathrm{mg} / \mathrm{dL}$. Alkaline phosphatase increased to $130 \mathrm{U} / \mathrm{L}$.

\section{Radiological findings}

Multiple pulmonary nodules were observed on the chest 
CT. PET/CT taken due to the re-aggravated back pain and an aggravation of existing bone metastasis and new bone lesions were observed in the skull base, spine, sternum, both scapulas, pelvis, and both femurs (Figure 3B).

\section{Treatment and progress}

After the patient was hospitalized, an intravenous injection of high dose corticosteroid (methylprednisolone, $2 \mathrm{mg} / \mathrm{kg}$ / day) was tried and stereotactic radiation therapy with $\mathrm{Cy}$ berKnife was administered to 5th lumbar spine metastasis. In addition, prophylactic radiotherapy was administered for weight bearing sight (both hip area) and skull base metastasis.

One month after high dose corticosteroid therapy, the patient continued to complain of back pain. Bone scan and CT scan revealed aggravation of pulmonary and bone metastasis. We concluded that corticosteroid was not effective and discontinued administration. For pain control, an additional stereotactic radiation therapy to second lumbar spine and sacrum was performed, and transforaminal 5th lumbar spine root block was undertaken. Afterwards, the patient newly complained of left arm tingling sensation, and T1 and T2 level cord compression was observed, and therefore, additional radiotherapy was administered for the cervical and thoracic spine. During this process, because radiation induced thrombocytopenia was observed, intermittent platelet transfusion was performed.

On June 2012, under circumstances where specific treatment plan could not be considered, celecoxib (1,000 mg/day), a selective cyclooxygenase-2 (COX-2) inihibitor, began to be tried. However, the patient newly began to complain of numbness in both legs, and deterioration of their motor grade, and voiding difficulty due to neurogenic bladder. Cervical and thoracic spine CT revealed severe cord compression at T8 and T11 level due to bone metastasis. Although surgery was considered, it was difficult to perform due to thrombocytopenia, and hence, stereotactic radiation therapy was administered. Nonetheless, the patient soon progressed to a state of paraplegia, and urinary catheter was inserted. CT scan showed pleural effusion, and liver, adrenal, and spleen metastasis. Approximately after 3 months of hospitalization, the patient was deceased on July 2012 due to disease progression despite conservative treatment.

\section{Discussion}

IMT was originally first reported in the lungs in $1937^{5}$. Since then, it has been reported in various sites such as mesentery, stomach, small intestines, large intestines, liver, mediastinum, retroperitoneum, and bladder. The most common organ the disease arises in is the lung, and the most common sites of extrapulmonary IMT include mesentery and omentum. IMT is defined as a tumor composed of differentiated myofibroblastic spindle cells usually accompanied by numerous plasma cells and lymphocytes. It is not certain if IMT is a reactive lesion or a true neoplasm. IMT was classified as an "intermediate malignancy (rarely metastasizing)" by the World Health Organization in $2002^{6}$ and the multiple metastasis of IMT has been very rarely reported. However, when looking at the report that chromosomal translocation involving $A L K$ gene was recently discovered to be present in $50 \%$ of IMTs, it suggests that approximately $50 \%$ of IMTs may have a malignant nature ${ }^{7}$. Complete surgical resection is the treatment of choice for IMTs not only to exclude malignancy but also to achieve a cure. The prognosis of patients who undergo radical resection of IMTs has been known to be favorable.

IMTs arising in the lungs are usually asymptomatic, and are discovered as a solitary nodule or mass by chest roentgenogram. It is typically located in the lung parenchyma but may appear in the trachea or the bronchi. Diagnosis of pulmonary IMT is known to be difficult to establish before surgery because of its diversified radiologic manifestations and because it can be difficult to distinguish from malignant tumors on small tissue samples obtained from bronchoscopic examination or needle biopsy. In other words, in most cases, accurate diagnosis can be given after resection. The prognosis of patients undergoing complete resection of the IMT arising in the lungs is known to be favorable ( 5 and 10 year survival rate, $91 \%$ and $77 \%$, respectively) ${ }^{1}$.

Surgical resection is a priority when feasable, but radiotherapy, anticancer chemotherapy, or administration of steroids or non-steroidal anti-inflammatory drugs has been employed for anatomically and functionally inoperable patients, and also in patients with recurrent diseases. However, the results of these treatments are widely variable, ranging from ineffective to complete regression. Regarding anticancer drugs, anthracyclins are sometimes helpful in IMTs originating from within the abdominal cavity ${ }^{2,3}$. In cases of IMTs originating from the lungs, anticancer drugs such as paclitaxel and carboplatin are sometimes helpful but in most cases, they are ineffective ${ }^{8,9}$. Interestingly, there are a few reports that non-steroidal antiinflammatory drugs or steroids have favorable effects in patients with positive vascular endothelial growth factor or COX2 compared with patients without either of them ${ }^{10}$. However, there is no report of its effect in the case of pulmonary IMTs. Recently, it has been reported that crizotinib, an ALK inhibitor, can be effective in some cases in which ALK is positive on immunohistochemisty staining ${ }^{7}$.

In this case study, there was a recurrence unlike most previous cases of IMTs originating from the lungs, which usually had no particular problems after surgery. Furthermore, bone metastasis progressed in a very aggressive manner, which has been very rarely reported up to the present. To the end, there was no observation of lymph node metastasis. While there were some reports that radiotherapy or treatment with 
non-steroidal anti-inflammatory drugs or steroids is effective against bone lesions of IMTs, this case progressed aggressively despite such treatments. In the case of this patient, there was no drugs proven to be effective, and anti-cancer treatment could not be performed due to his poor physical condition in which the disease progressed rapidly. Ultimately, the IMT progressed to the organs within the abdominal cavity including liver, spleen, and even adrenal glands.

Therefore, there is a need to recognize that even pulmonary IMTs can recur, cause aggressive bone metastasis and multiple metastasis to other organs, and may have an unfavorable prognosis. A close long term follow-up after surgery and not only CT scans but also tests such as alkaline phosphatase level, PET/CT, and bone scans will be helpful in discovering and predicting bone metastasis as well as metastasis to organs other than the lungs. For developing future treatment modalities and prognostic markers for IMTs originating from the lungs, accumulation of various cases of IMTs and molecular biological studies on them are needed.

\section{Acknowledgements}

This work is supported by a grant (50452-2013) from the Korea Institute of Radiological and Medical Sciences Research Fund (RTR).

\section{References}

1. Cerfolio RJ, Allen MS, Nascimento AG, Deschamps C, Trastek VF, Miller DL, et al. Inflammatory pseudotumors of the lung. Ann Thorac Surg 1999;67:933-6.

2. Hagenstad CT, Kilpatrick SE, Pettenati MJ, Savage PD. Inflammatory myofibroblastic tumor with bone marrow involve- ment: a case report and review of the literature. Arch Pathol Lab Med 2003;127:865-7.

3. Kim DY, Park HS, Kim SM, Park JH, Hong YS, Lee JL, et al. Inflammatory myofibroblastic tumor showing durable remission after anthracycline-containing cytotoxic chemotherapy: report of a case. Korean J Med 2012;82:749-53.

4. Sasagawa Y, Akai T, Itou S, Iizuka H. Multiple intraosseous inflammatory myofibroblastic tumors presenting with an aggressive clinical course: case report. Neurosurgery 2011;69:E1010-5.

5. Brunn H. Two interesting benign lung tumors of contradictory histopathology. J Thorac Surg 1939;9:119-31.

6. Coffin CM, Fletcher JA. Inflammatory myofibroblastic tumor. In: Fletcher CD, Unni KK, Mertens F, editors. World Health Organization classification of tumours: pathology and genetics of tumours of soft tissue and bone. Lyon: IARC Press; 2002. p. 91-3.

7. Coffin CM, Patel A, Perkins S, Elenitoba-Johnson KS, Perlman E, Griffin CA. ALK1 and p80 expression and chromosomal rearrangements involving 2p23 in inflammatory myofibroblastic tumor. Mod Pathol 2001;14:569-76.

8. Suetsugu S, Yamamoto $\mathrm{H}$, Izumi M, Takayama K, Inoue $\mathrm{H}$, Nakanishi Y. A case of rapidly growing inflammatory myofibroblastic tumor in the lung. Nihon Kokyuki Gakkai Zasshi 2009;47:1156-60.

9. Kubo N, Harada T, Anai S, Otsubo K, Yoneshima Y, Ijichi K, et al. Carboplatin plus paclitaxel in the successful treatment of advanced inflammatory myofibroblastic tumor. Intern Med 2012;51:2399-401.

10. Applebaum H, Kieran MW, Cripe TP, Coffin CM, Collins $\mathrm{MH}$, Kaipainen A, et al. The rationale for nonsteroidal antiinflammatory drug therapy for inflammatory myofibroblastic tumors: a Children's Oncology Group study. J Pediatr Surg 2005;40:999-1003. 